\title{
Risk of deep vein thrombosis and pulmonary embolism in asthma
}

\author{
Christof J. Majoor ${ }^{1}$, Pieter W. Kamphuisen², Aeilko H. Zwinderman³, \\ Anneke ten Brinke ${ }^{4}$, Marijke Amelink', Lucia Rijssenbeek-Nouwens ${ }^{5}$, \\ Peter J. Sterk' Harry R. Büller ${ }^{6}$ and Elisabeth H. Bel ${ }^{1}$

\begin{abstract}
Affiliations:
'Dept of Respiratory Medicine, Academic Medical Centre, Amsterdam,

${ }^{2}$ Dept of Vascular Medicine, University Medical Centre Groningen, Groningen,

${ }^{3}$ Dept of Clinical Epidemiology and Biostatistics, Academic Medical Centre, Amsterdam,

${ }^{4}$ Dept of Respiratory Medicine, Medisch Centrum Leeuwarden, Leeuwarden, and

${ }^{6}$ Dept of Vascular Medicine, Academic Medical Centre, Amsterdam, The Netherlands.

${ }^{5}$ Dutch Asthma Centre, Davos Dorf, Switzerland.
\end{abstract}

\section{Correspondence:}

C.J. Majoor, Dept. of Respiratory Medicine, Academic Medical Center, PO Box 22660, 1100 DE Amsterdam, The Netherlands.

E-mail: c.j.majoordamc.uva.nl

ABSTRACT Increasing evidence suggests that patients with asthma have activated coagulation within the airways. Whether this leads to an increase in venous thromboembolic events is unknown. We therefore assessed the incidence of venous thromboembolic events in patients with mild-to-moderate and severe asthma as compared with an age- and sex-matched reference population.

648 patients with asthma (283 with severe and 365 patients with mild-to-moderate asthma) visiting three Dutch outpatient asthma clinics were studied. All patients completed a questionnaire about a diagnosis of deep vein thrombosis and pulmonary embolism in the past, their risk factors, history of asthma and medication use. All venous thromboembolic events were objectively verified.

In total, 35 venous thromboembolic events (16 deep vein thrombosis and 19 pulmonary embolism) occurred at a median age of 39 (range 20-63) years. The incidence of pulmonary embolism in patients with severe asthma was 0.93 (95\% CI $0.42-1.44)$ per 1000 person-years, 0.33 (95\% CI $0.07-0.60$ ) in mild-tomoderate asthma and 0.18 (95\% CI 0.03-0.33) in the general population, respectively. Severe asthma and oral corticosteroid use were independent risk factors of pulmonary embolism (hazard ratios 3.33 (1.169.93) and 2.82 (1.09-7.30), respectively). Asthma was not associated with deep vein thrombosis.

Severe asthma greatly enhances the risk of pulmonary embolism, particularly if chronic corticosteroids are used.

@ERSpublications

Severe asthma greatly enhances the risk of pulmonary embolism, particularly if chronic corticosteroids are used http://ow.ly/moNaO

This article has supplementary material available from www.erj.ersjournals.com

Received: Sept 212012 | Accepted after revision: Nov 212012 | First published online: Dec 202012

Support statement: The study was funded by a grand from Dutch Asthma Foundation (grant number AF 3.2.11.021).

Conflict of interest: Disclosures can be found alongside the online version of this article at www.erj.ersjournals.com

Copyright @ERS 2013 


\section{Introduction}

Chronic inflammatory diseases have been associated with activation of coagulation $[1,2]$ and an increased risk of venous thromboembolic events (VTEs), in particular during active disease. This has been shown for inflammatory bowel disease [3], rheumatoid arthritis [4], diabetes mellitus [5] and chronic obstructive pulmonary disease (COPD) [6]. Asthma has also been associated with a procoagulant and antifibrinolytic activity in the airways $[7,8]$. However, whether this procoagulant shift translates into a higher risk of symptomatic VTE is unknown.

Therefore, we hypothesised that asthma, in particular severe, refractory asthma [9], predisposes to an increased risk of thromboembolic complications. The present study was designed to determine the incidence of deep vein thrombosis (DVT) and pulmonary embolism (PE) in outpatients with mild-to-moderate and severe asthma, and to compare the incidence rates to an age- and sex-matched reference population.

\section{Methods}

Study population

Adult patients with mild-to-moderate or severe asthma according to international criteria [10, 11], who visited the outpatient pulmonary clinic of three Dutch tertiary asthma clinics (one academic, one nonacademic and one asthma centre) were consecutively recruited between December 1, 2010 and May 1, 2011. The reference population was retrieved from a publication by NAESs et al. [12] representing a sample from the general population in Norway as this population is the best match to the Dutch population. This population of 94194 individuals aged $\geqslant 20$ years (mean age 46 years (range 20-103 years)) participated in a large-scale general health study (the HUNT2 study) and were followed from 1995-2001 [13].

\section{Questionnaire}

To assess the prevalence of VTE, subjects completed a questionnaire during their routine follow-up visit to the outpatient clinic. The questionnaire contained a set of questions about a history of DVT and PE, anticoagulant therapy and risk factors for VTE, as well as asthma-specific questions, including inhaled or oral corticosteroid treatment at the time of the thromboembolic event. The complete questionnaire is given in the online supplementary material (fig. S1).

\section{Diagnosis of VTE}

In the study population, all VTEs were objectively verified. In all identified events, medical records of the patients were reviewed and adjudicated blindly using international criteria for DVT and PE [14-16]. In the reference population, NAESs et al. [12] verified VTEs by using the same criteria. Additional details on the method for verifying the diagnosis of PE and DVT are provided in the online supplementary material.

VTE was categorised as first event or recurrent event, as well as provoked or idiopathic event. Provoked VTE was defined as VTE occurring under oral contraceptive use, recent surgery, confinement to bed, positive family history of VTE and pregnancy. Other VTEs were considered idiopathic.

\section{Statistical analysis}

The primary study outcome was the incidence of first episode of DVT and PE in asthma patients. Secondary outcomes were covariables associated with the incidence of first DVT and PE. Cumulative incidences of VTE, PE and DVT were estimated using Kaplan-Meier survival probabilities. In order to match the incidences of the present population with those of the reference population we performed an indirect standardisation procedure using age- and sex-specific VTE incidence numbers from NAESs et al. [12]. Using these numbers, the expected cumulative PE hazard at the age of the first episode of PE (or at the age of censoring if a patient did not have PE), was calculated for every patient by summing the age-sex-specific incidences of NAESS et al. [12]. The expected cumulative PE hazards after asthma onset were calculated as the differences between the total cumulative hazards minus the cumulative hazards until the age of asthma onset.

The incidence of PE in the age- and sex-matched general population was calculated as the expected number of $\mathrm{PE}$ according to NAESS et al. [12] divided by the total number of person-years for our study population. Rate ratios were computed by dividing the number of PE in our sample by the sum of the expected cumulative PE hazards. Exact 95\% confidence intervals were calculated assuming that the number of PEs followed a Poisson distribution. The same procedure was performed to calculate the incidences and rate ratios of DVT.

Multivariate regression of the PE or DVT risk on cofactors was performed using the Cox proportional hazards regression model. Age-sex-specific population hazards (according to NAEss et al. [12]) were used as time-dependent offset-variables, as described by ANDERSON et al. [17]. Asthma severity was the timedependent covariate. Potential cofactors were atopy, sex, body mass index (BMI), smoking status, inhaled corticosteroid use, oral corticosteroid use and forced expiratory volume in $1 \mathrm{~s} \mathrm{(FEV1)} \mathrm{relative} \mathrm{to} \mathrm{forced} \mathrm{vital}$ capacity (FVC). Data were processed in SPSS version 18.0 (SPSS Inc., Chicago, IL, USA). 


\section{Results}

A total of 762 adults with asthma fulfilled the criteria for inclusion. 13 patients were irretrievable, 91 did not return the questionnaire, six patients were excluded for psychological reasons, three had language problems and, in one patient, demographic data was incomplete (fig. 1). Thus, a total of 648 patients, 365 patients with mild-to-moderate asthma and 283 patients with severe asthma, were analysed. Patients were aged between 18 and 88 years. Other patient characteristics are summarised separately for the two groups in table 1. Characteristics of nonparticipating patients did not differ from those participating (data not shown).

VTEs in patients with asthma

35 (5.4\%) first episodes of VTE occurred in patients with asthma (17 with mild-to-moderate asthma and 18 with severe asthma) during 31889 person-years (table 2). 16 (46\%) patients suffered from DVT and 19 (54\%) from PE (either with or without DVT). Definite DVT was confirmed in 12 patients, and four patients had a probable DVT (clinical suspicion without objective diagnostic imaging). Definite PE was diagnosed in 16 patients ( 11 by computed tomography scan and five by high probability ventilation/perfusion (V/Q) scan) and three patients had a probable PE (one with typical symptoms and intermediate probability V/Q scan, and two with typical symptoms and proven DVT). All patients (with both definite and probable DVT and PE) were treated for 6 months with anticoagulation therapy. Therefore, probable cases were included in the analysis.

VTE occurred in $10(3.8 \%)$ out of 648 males and $25(6.5 \%)$ out of 648 females $(p=0.04)$. Provoked VTE occurred in $24(68.6 \%)$ patients (oral contraceptive use, $n=11$; recent surgery, $n=9$; positive family history of VTE, $n=6$; and pregnancy, $n=3$ ). Seven patients had more than one risk factor. Although not significant, idiopathic VTE was noted more often in patients with severe asthma (39\% versus $24 \%$; $=0.31$ ).

The median age at the first event was 39 years (range 20-57 years) in patients with severe asthma and 39 years (range 22-63 years) in patients with mild-to-moderate asthma. In patients with severe asthma, the majority of VTEs (89\%) occurred after the onset of asthma, as compared with $41 \%$ in patients with mild-tomoderate asthma (Chi-squared $\mathrm{p}=0.003)$. Seven $(39 \%)$ out of 18 patients with severe asthma and VTE and three $(18 \%)$ out of 17 patients with mild-to-moderate asthma had recurrent thromboembolic events $(\mathrm{p}=0.16)$. Twelve $(67 \%)$ out of 18 patients with severe asthma and VTE and none with mild-to-moderate asthma used chronic oral corticosteroids at the time of the first VTE.

\section{Comparison between patients with severe asthma, mild-to-moderate asthma and the general population}

The cumulative incidences for VTE, PE and DVT for patients with severe and mild-to-moderate asthma and the general population are shown in figure 2. The incidence of VTE in patients with severe asthma was 1.29 per 1000 person-years (95\% CI 0.69-1.88) compared with 0.95 (95\% CI 0.50-1.40) in patients with

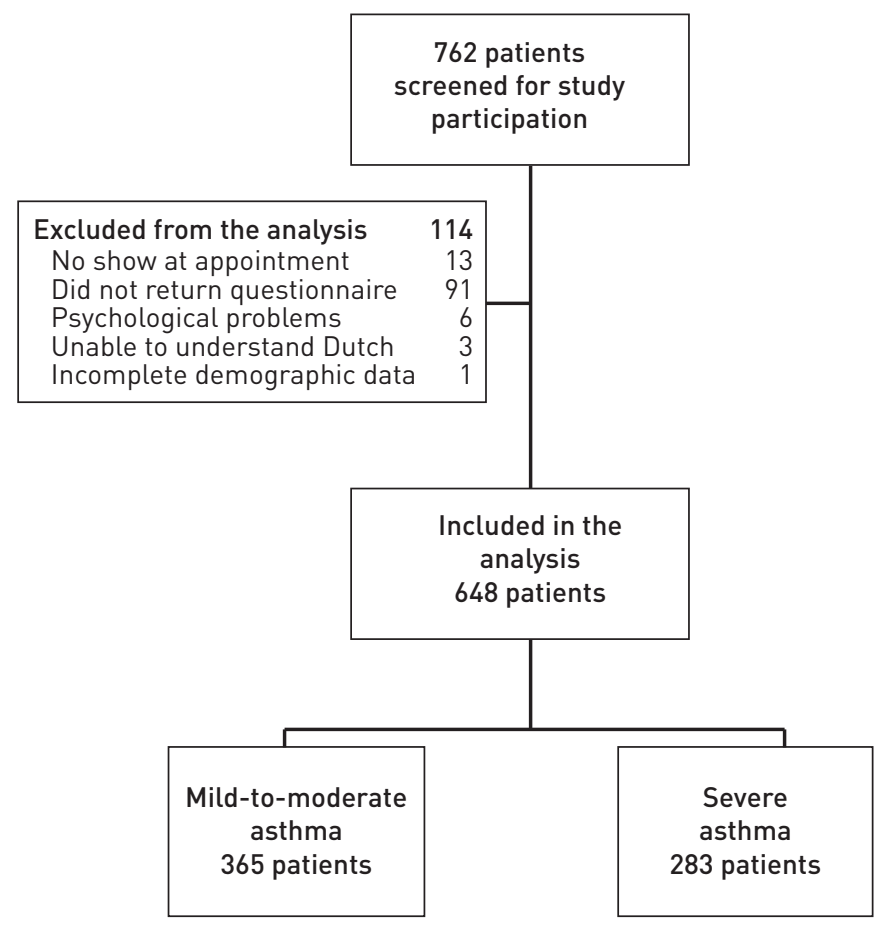




\section{TABLE 1 Baseline characteristics}

Mild-to-moderate asthma

Patients $\mathbf{n}$
Age years
Female sex
BMI kg· $\mathbf{m}^{-2}$
Age of asthma onset years $\#$
Duration of asthma years ${ }^{\#}$
Atopy
Smoking history
Never-smoker
Ex-smoker
Current smoker
Severe exacerbations in previous
$<1$ per year
$1-2$ per year
$>2$ per year
FEV1 post-bronchodilator \% pred
ICS dose $\geqslant 1000 \mu \mathrm{g} \cdot$ day $^{-1}$
Chronic oral corticosteroid
Omalizumab

Severe asthma

$\begin{array}{cc}365 & 283 \\ 50(18-88) & 51(17-77) \\ 58 & 61 \\ 28(17-45) & 27(18-59) \\ 39(0-78) & 27(0-70) \\ 5.0(0.1-78) & 20(0.1-76) \\ 42 & 49 \\ 52 & \\ 40 & 64 \\ 8 & 33 \\ 73 & 3 \\ 19 & 28 \\ 8 & 31 \\ 97.0(22-146) & 41 \\ 0 & 84.9(23.1-133) \\ 0 & 85 \\ 0 & 38 \\ & 7\end{array}$

Data are presented as median (range) or \%, unless otherwise stated. BMI: body mass index; FEV1: forced expiratory volume in $1 \mathrm{~s}$; \% pred: \% predicted; ICS: inhaled corticosteroid. \# : data derived from questionnaires; not objectively confirmed in 26 patients.

mild-to-moderate asthma and 0.46 (95\% CI $0.23-0.70)$ in the general population, respectively. The incidence of PE in patients with severe asthma was 0.93 (95\% CI $0.42-1.44)$ per 1000 person-years, 0.33 (95\% CI 0.07-0.60) in patients with mild-to-moderate asthma and 0.18 (95\% CI $0.03-0.33)$ in the general population, respectively. The incidence of DVT in patients with severe asthma was 0.36 (95\% CI 0.04-0.67) per 1000 person-years, 0.61 (95\% CI $0.25-0.98)$ in patients with mild-to-moderate asthma and $0.28(95 \%$ CI $0.10-0.47)$ in the general population, respectively.

Standardised rate ratios for first PE and DVT in patients with severe and mild-to-moderate asthma were 8.93 (95\% CI 4.62-15.63) and 3.97 (95\% CI 0.97-9.12), respectively, while the rate ratios for DVT were not significantly increased (1.62 (95\% CI $0.44-4.14)$ and 1.45 (95\% CI $0.30-4.23)$, respectively). Excluding the

\section{TABLE 2 Venous thromboembolic events (VTEs) in study population}

Mild-to-moderate asthma

\begin{tabular}{lcc}
\hline & & 283 \\
Patients & 365 & 13975 \\
Total number of person-years & 17914 & 18 \\
Before and after asthma onset & 17 & 5 \\
All VTEs & 11 & 13 \\
Only DVT & 6 & 2 \\
PE \pm DVT & & 1 \\
Before asthma onset & 10 & 1 \\
All VTEs & 8 & \\
Only DVT & 2 & 16 \\
PE \pm DVT & \multicolumn{2}{c}{} \\
After onset of asthma & 7 & 4 \\
All VTEs & 3 & 12 \\
Only DVT & 4 & \\
PE \pm DVT & 4 & \\
\hline
\end{tabular}

Data are presented as n. DVT: deep venous thrombosis; PE: pulmonary embolism. 

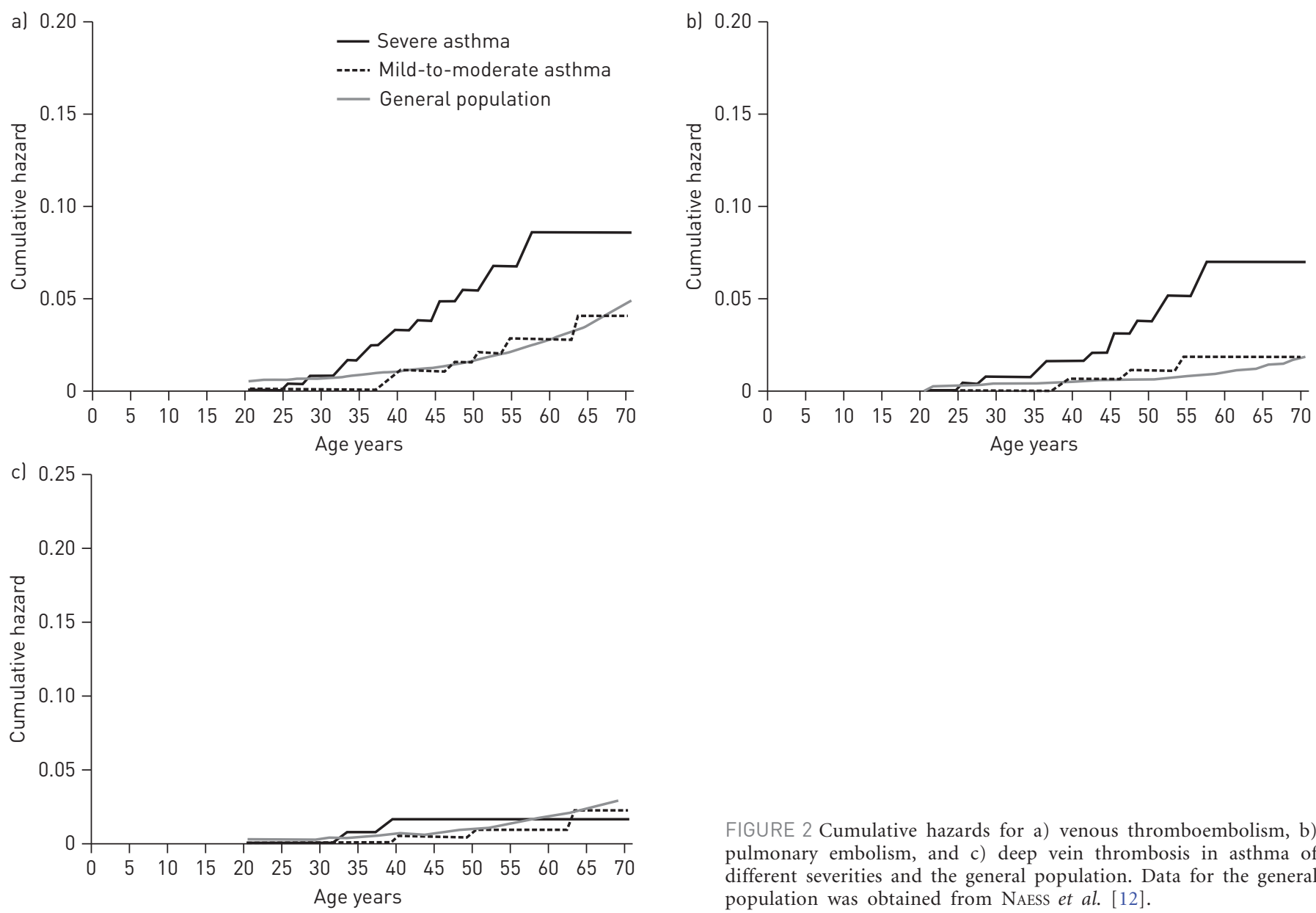

FIGURE 2 Cumulative hazards for a) venous thromboembolism, b) pulmonary embolism, and c) deep vein thrombosis in asthma of different severities and the general population. Data for the general population was obtained from NAESs et al. [12].

probable cases of DVT and PE, the rate ratios did not markedly change the results: the rate ratio for PE was 8.93 (95\% CI 4.62-15.63) and 2.67 (95\% CI 0.55-7.80), respectively (table 3).

Risk factors of DVT and PE

Univariate Cox regression showed that severe asthma and the use of oral corticosteroids were associated with PE. Mild-to-moderate asthma, severe asthma and BMI were associated with DVT. Other factors, such as inhaled corticosteroid dose (for both as a continuous variable and a dichotomous variable with a cut-off dose of fluticasone, or equivalent drug, of $1000 \mu \mathrm{g} \cdot \mathrm{day}^{-1}$ ), atopy, being a current or ex-smoker, sex and FEV1/FVC were not associated with PE or DVT. In the multivariate Cox regression model, only severe asthma (hazard ratio 3.33, 95\% CI 1.16-9.93) and oral corticosteroid use (hazard ratio 2.82, 95\% CI 1.097.30) were associated with PE, and BMI with DVT (hazard ratio 1.09, 95\% CI 1.01-1.16).

TABLE 3 Rate ratios for first pulmonary embolism (PE) and deep vein thrombosis (DVT) events in our asthma population versus the general population

Mild-to-moderate asthma

\begin{tabular}{lcc}
\hline Patients $\mathbf{n}$ & 365 & 283 \\
All VTEs (definite and probable) & & $1.62(0.44-4.14)$ \\
DVT & $1.45(0.30-4.23)$ & $8.93(4.62-15.63)$ \\
PE & $3.56(0.97-9.12)$ & $1.21(0.25-3.55)$ \\
All definite VTEs & & $8.93(4.62-15.63)$ \\
$\quad$ DVT & $1.45(0.30-4.23)$ & $2.67(0.55-7.80)$ \\
PE
\end{tabular}

Data are presented as rate ratio $(95 \% \mathrm{CI})$, unless otherwise stated. VTE: venous thromboembolic event. 


\section{Discussion}

Our results show an almost nine-fold increased risk of PE in patients with severe asthma as compared to the general population. In addition, a trend towards a 3.5-fold increased risk of PE was found in patients with mild-to-moderate asthma. Severe asthma and the use of oral corticosteroids were risk factors of PE in multivariate analysis. Interestingly, asthma was not associated with DVT. These results suggest that the incidence of $\mathrm{PE}$ is increased in patients with severe asthma, particularly in patients using oral corticosteroids.

To our knowledge this is the first study to investigate the relationship between asthma of different severities and VTEs. Two studies in the primary care setting found an association between VTE and asthma [18, 19]. In the first study [18], a slightly increased risk of PE was found in patients with asthma, whereas in the second study [19], the prevalence of asthma in females using oral contraceptives who were diagnosed with VTE was somewhat higher compared with those without VTE [19]. In both studies, the severity and treatment of asthma were not explored. Another study evaluated the incidence of VTE in patients with atopic diseases and found a higher risk of DVT and PE in patients with allergic rhinitis or elevated specific immunoglobulin E to a panel of common aeroallergens. No increased risk was found in patients with asthma, probably because the sample size was too small [20]. Yet another study found an association between Churg-Strauss syndrome and DVT, which is interesting as Churg-Strauss syndrome is often associated with severe asthma [21]. Also, other airway diseases, such as COPD, have been associated with increased risk of $\mathrm{PE}$, particularly among hospitalised patients during an exacerbation [6, 22]. Our results show that patients with asthma are at increased risk of PE, in particular those with severe disease. Together, these studies suggest that disease severity is an important risk factor for PE in patients with airways disease.

The strength of our study is the high number of patients $(n=283)$ with well-characterised severe asthma [10], which is comparable to the number of patients included in the National Institutes of Healthsponsored multicentre Severe Asthma Research Programme (SARP) studies [23, 24]. Nevertheless, the number of patients with PE and DVT in our study was modest; hence some of the subanalyses should be interpreted with caution. This could also explain the lack of association between PE and mild-to-moderate asthma. Furthermore, our study was based on self-reported VTE, which might have caused misclassification. Overestimation was, however, ruled out as all self-reported thromboembolic events were confirmed by objective measurements including compression ultrasound for DVT and computed tomography scanning or high probability ventilation/perfusion scanning for PE.

Finally, the Norwegian population was used as control group for Dutch asthma patients. Observed incidence rates of VTE in the Norwegian population were similar to rates for first events in other western European countries, including France and Sweden $[25,26]$. Therefore, we believe that the Norwegian population could be used as an adequate reference population in our study.

The mechanism by which severe asthma may predispose to PE is complex. First, there is extensive evidence that inflammation alters the balance between the procoagulant and fibrinolytic activity, and that inflammation and coagulation stimulate each other $[8,27]$. This has been observed in animal models, in the lung tissue of humans with acute lung injury [28] and in patients with pneumonia [29]. Also, there is evidence of activation of the extrinsic coagulation cascade in the asthmatic airway, with involvement of both plasma and locally derived factors $[7,8]$. This is supported by a recent finding showing local vascular inflammation in asthma and COPD [30], as well as the observation of a higher incidence of PE than DVT in COPD patients [22], and evidence that in other chronic inflammatory disorders, such as diabetes, rheumatoid arthritis and ulcerative colitis, a higher incidence of PE has not been observed [3-5]. Taken together, the results of the present study suggest that, particularly in severe asthma, the balance between coagulation and fibrinolysis is seriously disturbed.

Secondly, inactivity of the patients with severe asthma might be a potential trigger for VTE. However, this would be expected to lead to a higher incidence of DVT than PE, which was not the case in our study.

Finally, patients with severe asthma continuously use (ultra)high doses of inhaled corticosteroids, receive bursts of systemic corticosteroid during exacerbations, and often need chronic oral corticosteroid treatment for control of their asthma. Corticosteroid-induced hypercoagulability has been described for many years [31], but it is still controversial whether the use of corticosteroids themselves or the underlying (severe) disease contributes to the hypercoagulable state [32].

The findings of the present study may have important clinical implications. PE is a potentially lifethreatening complication that may occur in relatively young patients with severe asthma. Doctors should therefore increase their awareness and lower the threshold for the evaluation of patients with severe asthma for possible PE. In addition, we believe that strategies to reduce the risk of PE, such as thromboprophylaxis, may be considered in patients with prednisone-dependent asthma.

In conclusion, our study suggests that patients with asthma, in particular those with severe, refractory disease, have a high risk of PE, which may be further increased by asthma severity and oral corticosteroids. 


\section{Acknowledgements}

We thank J. Christa de Groot (Dept of Respiratory Medicine, MC Leeuwarden, Leeuwarden, The Netherlands) and Selma B. de Nijs (Dept of Respiratory Medicine, Academic Medical Centre, Amsterdam, The Netherlands) for their help with the recruitment of the patients.

\section{References}

1 Del Rosso M, Fibbi G, Matucci Cerinic M. The urokinase-type plasminogen activator system and inflammatory joint diseases. Clin Exp Rheumatol 1999; 17: 485-498.

2 Saibeni S, Saladino V, Chantarangkul V, et al. Increased thrombin generation in inflammatory bowel diseases. Thromb Res 2010; 125: 278-282.

3 Grainge MJ, West J, Card TR. Venous thromboembolism during active disease and remission in inflammatory bowel disease: a cohort study. Lancet 2010; 375: 657-663.

4 Matta F, Singala R, Yaekoub AY, et al. Risk of venous thromboembolism with rheumatoid arthritis. Thromb Haemost 2009; 101: 134-138.

5 Stein PD, Goldman J, Matta F, et al. Diabetes mellitus and risk of venous thromboembolism. Am J Med Sci 2009; 337: 259-264.

6 Rizkallah J, Man SF, Sin DD. Prevalence of pulmonary embolism in acute exacerbations of COPD: a systematic review and metaanalysis. Chest 2009; 135: 786-793.

7 Brims FJ, Chauhan AJ, Higgins B, et al. Coagulation factors in the airways in moderate and severe asthma and the effect of inhaled steroids. Thorax 2009; 64: 1037-1043.

8 de Boer JD, Majoor CJ, van 't Veer C, et al. Asthma and coagulation. Blood 2012; 119: 3236-3244.

9 ten Brinke A, Zwinderman AH, Sterk PJ, et al. "Refractory" eosinophilic airway inflammation in severe asthma: effect of parenteral corticosteroids. Am J Respir Crit Care Med 2004; 170: 601-605.

10 Bel EH, Sousa A, Fleming L, et al. Diagnosis and definition of severe refractory asthma: an international consensus statement from the Innovative Medicine Initiative (IMI). Thorax 2011; 66: 910-917.

11 National Institutes of Health, Heart, Lung, and Blood Institute. Global initiative for asthma. Global strategy for asthma management and prevention. NHLBI/WHO Workshop Report, 2010. http://ginasthma.org/local/uploads/ files/GINA_Report_March13.pdf Date last accessed: July 1, 2013.

12 Naess IA, Christiansen SC, Romundstad P, et al. Incidence and mortality of venous thrombosis: a population-based study. J Thromb Haemost 2007; 5: 692-699.

13 Holmen J, Midthjell K, Krüger Ø, et al. The Nord-Trøndelag health study 1995-1997 (HUNT2): objectives, contents, methods and participation. Norsk Epidemiologi 2003; 13: 19-32.

14 Gottschalk A, Sostman HD, Coleman RE, et al. Ventilation-perfusion scintigraphy in the PIOPED study. Part II. Evaluation of the scintigraphic criteria and interpretations. J Nucl Med 1993; 34: 1119-1126.

15 Cushman M, Tsai AW, White RH, et al. Deep vein thrombosis and pulmonary embolism in two cohorts: the longitudinal investigation of thromboembolism etiology. Am J Med 2004; 117: 19-25.

16 van Belle A, Büller HR, Huisman MV, et al. Effectiveness of managing suspected pulmonary embolism using an algorithm combining clinical probability, D-dimer testing, and computed tomography. JAMA 2006; 295: 172-179.

17 Andersen PK, Borch-Johnsen K, Deckert T, et al. A Cox regression model for the relative mortality and its application to diabetes mellitus survival data. Biometrics 1985; 41: 921-932.

18 Huerta C, Johansson S, Wallander MA, et al. Risk factors and short-term mortality of venous thromboembolism diagnosed in the primary care setting in the United Kingdom. Arch Intern Med 2007; 167: 935-943.

19 Farmer RD, Lawrenson RA, Todd JC, et al. Oral contraceptives and venous thromboembolic disease. Analyses of the UK General Practice Research Database and the UK Mediplus database. Hum Reprod Update 1999; 5: 688-706.

20 Undas A, Ciesla-Dul M, Drazkiewicz T, et al. Association between atopic diseases and venous thromboembolism: a case-control study in patients aged 45 years or less. J Thromb Haemost 2011; 9: 870-873.

21 Allenbach Y, Seror R, Pagnoux C, et al. High frequency of venous thromboembolic events in Churg-Strauss syndrome, Wegener's granulomatosis and microscopic polyangiitis but not polyarteritis nodosa: a systematic retrospective study on 1130 patients. Ann Rheum Dis 2009; 68: 564-567.

22 Bertoletti L, Quenet S, Mismetti P, et al. Clinical presentation and outcome of venous thromboembolism in COPD. Eur Respir J 2012; 39: 862-868.

23 Moore WC, Meyers DA, Wenzel SE, et al. Identification of asthma phenotypes using cluster analysis in the Severe Asthma Research Program. Am J Respir Crit Care Med 2010; 181: 315-323.

24 Wenzel SE, Busse WW. Severe asthma: lessons from the Severe Asthma Research Program. J Allergy Clin Immunol 2007; 119: 14-21.

25 Oger E. Incidence of venous thromboembolism: a community-based study in Western France. EPI-GETBP Study Group. Groupe d'Etude de la Thrombose de Bretagne Occidentale. Thromb Haemost 2000; 83: 657-660.

26 Zöller B, Li X, Sundquist J, et al. Age- and gender-specific familial risks for venous thromboembolism: a nationwide epidemiological study based on hospitalizations in Sweden. Circulation 2011; 124: 1012-1020.

27 Levi M, van der Poll T. Inflammation and coagulation. Crit Care Med 2010; 38: Suppl. 2, S26-S34.

28 Hofstra JJ, Schouten M, Levi M. Thrombophilia and outcome in severe infection and sepsis. Semin Thromb Hemost 2007; 33: 604-609.

29 Smeeth L, Cook C, Thomas S, et al. Risk of deep vein thrombosis and pulmonary embolism after acute infection in a community setting. Lancet 2006; 367: 1075-1079.

30 Wanner A, Mendes ES. Airway endothelial dysfunction in asthma and chronic obstructive pulmonary disease: a challenge for future research. Am J Respir Crit Care Med 2010; 182: 1344-1351.

31 Cosgriff SW, Diefenbach AF, Vogt W Jr. Hypercoagulability of the blood associated with ACTH and cortisone therapy. Am J Med 1950; 9: 752-756.

32 van Zaane B, Nur E, Squizzato A, et al. Systematic review on the effect of glucocorticoid use on procoagulant, anticoagulant and fibrinolytic factors. J Thromb Haemost 2010; 8: 2483-2493. 